PATHOLOGY

MEDICAL RESEARCH

METHODS

Received

21 March 2012

Accepted

11 May 2012

Published

31 May 2012

Correspondence and requests for materials should be addressed to C.G. (cinzia. giannini@ic.cnr.it)

\section{OPEN \\ OPEN \\ SUBJECT AREAS: \\ IMAGING \\ Correlative Light and Scanning X-Ray Scattering Microscopy of Healthy and Pathologic Human Bone Sections}

\author{
C. Giannini' , D. Siliqi' , O. Bunk ${ }^{3}$, A. Beraudi², M. Ladisa' , D. Altamura' ', S. Stea ${ }^{2} \&$ F. Baruffaldi²
}

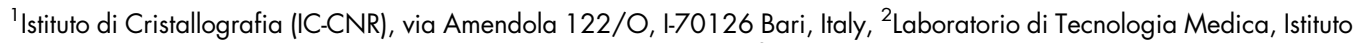
Ortopedico Rizzoli, Via di Barbiano 1/10, 40136 - Bologna, Italy, ${ }^{3}$ Paul Scherrer Institut, Swiss Light Source, 5232 Villigen PSI, Switzerland.

Scanning small and wide angle X-ray scattering (scanning SWAXS) experiments were performed on healthy and pathologic human bone sections. Via crystallographic tools the data were transformed into quantitative images and as such compared with circularly polarized light (CPL) microscopy images. SWAXS and CPL images allowed extracting information of the mineral nanocrystalline phase embedded, with and without preferred orientation, in the collagen fibrils, mapping local changes at sub-osteon resolution. This favorable combination has been applied for the first time to biopsies of dwarfism syndrome and Paget's disease to shed light onto the cortical structure of natural bone in healthy and pathologic sections.

one is a natural material with a hierarchical architecture of organic and inorganic structures organized at several different length scales ${ }^{1-4}$, working in concert to perform diverse mechanical, biological and chemical functions such as structural support, protection and storage of healing cells, and mineral ion homeostasis. A typical length scale for the basic bone unit, the osteon, is $100 \mu \mathrm{m}$. Each osteon is formed by concentric lamellae, approx. $10 \mu \mathrm{m}$ thick, composed of collagen fibrils that are on the order of $1 \mu \mathrm{m}$ wide. The latter consist of an inhomogeneous matrix of cross-linked type I collagen molecules, about 300 and $1.5 \mathrm{~nm}$ in length and diameter, respectively, mineralized with nanocrystalline carbonated hydroxyapatite (HA). Type I collagen and HA nanocrystals are therefore the basic building blocks of bone at the nanoscale (1-300 nm). Small-angle X-ray scattering (SAXS) is sensitive to changes of the refractive index at the nanoscale, here mainly due to the mineral nanocrystals embedded in collagen fibrils ${ }^{5,6}$; whereas wide-angle X-ray scattering (WAXS) is the interference pattern of the secondary waves scattered by the atomic electron density distribution within the crystallographic structure of the nanocrystals ${ }^{7}$. Circularly polarized light (CPL) microscopy is an imaging technique used to classify osteons in terms of collagen fiber orientation ${ }^{8}$.

Among the bone disorders ${ }^{9,10}$, Paget's disease is also called osteitis deformans. It is a chronic disorder with alterations of the bone remodeling equilibrium and accelerated deposition of not well-organized lamellar bone, in particular for trabecular bone. The result are enlarged and deformed bones, the so called radiographic mosaic bone pattern, causing pain at the main joints. Paget's disease might be caused by genetic factors in combination with viral exposure. Dwarfism is a medical condition that results in the underdevelopment of the body. It might be caused by genetic factors, nutritional, or hormone deficiencies.

In the present paper, we show how a detailed level of morphological and structural information can be gained by collecting both SAXS and WAXS data (SWAXS) in scanning mode with an X-ray spot in the micrometer range, to map the nanoscale bone architecture, at sub-osteon resolution, across an area of several square millimeters. Neck femur slices have been obtained from three different patients, subject to total hip arthroplasty, two of them being affected by Paget's disease and osteochondrodysplasic non-pituitary dwarfism, respectively, and one unaffected by any known musculoskeletal disease, but surgically treated for traumatic femur bone fracture. By means of robust crystallographic analytic methods ${ }^{11,17}$ the acquired scanning SWAXS data were converted into direct images of the size, shape and orientation of the mineral nanocrystals and directly compared with CPL images. This combination of SWAXS and CPL microscopy allowed us to image the complex architecture of the cross-linked type I collagen fibrils mineralized with carbonated HA nanocrystals. The nanometer ordering within the fibrils and the micrometer scale ordering in the stacking of the fibrils were studied as well as the distribution of the unoriented mineral phase. 


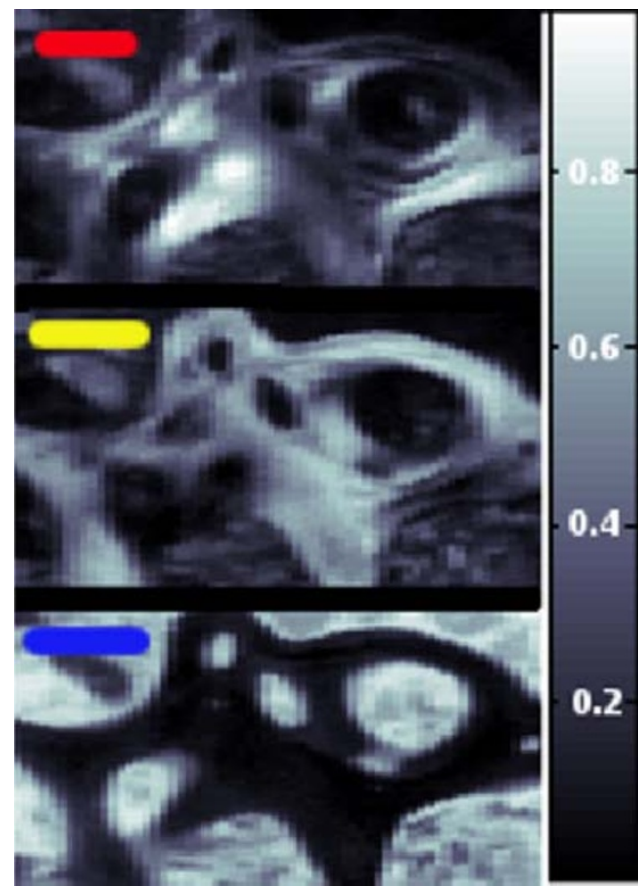

Figure 1 | Healthy biopsy: CCA analysis performed model by model. The correlation degree varies between 0 (black) and 1 (white).

\section{Results}

The femoral head sections from patients suffering from Paget's disease, dwarfism and no musculoskeletal disease have been investigated in identical areas using WAXS, SAXS, and CPL. The flow chart in Fig. S1 shows the data collection and reduction procedure for the scanning WAXS and SAXS data. The least correlated, i.e. principal WAXS signals were identified (Fig. S2a) using an 'adaptive binning' criterion ${ }^{12}$ and analyzed with a Rietveld-based program ${ }^{13}$, yielding, e.g., the dimensions of the mineral platelets. The radius of gyration calculated from these dimensions was found to be in excellent agreement with the one determined as a cross-check from the corresponding SAXS data. Using a canonical correlation analysis $(\mathrm{CCA})^{12,16,17}$ the contribution of three major principal signals was determined for each point of the scanning WAXS data. An example is shown in Fig. 1, where the top and center plot show different

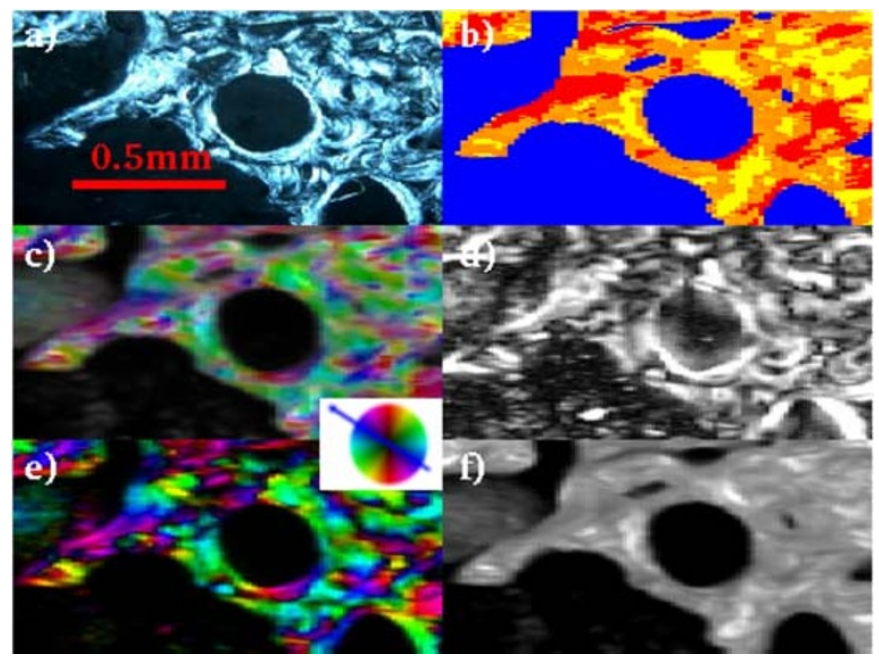

Figure $3 \mid$ CPL (a), WAXS (b), SAXS (c,d,e,f) analyses for the investigated Paget's disease biopsy ( 0.5 is the maximum degree of orientation). The colored wheel refers to the SAXS orientation map panels $\mathbf{c}$ and $\mathbf{e}$.

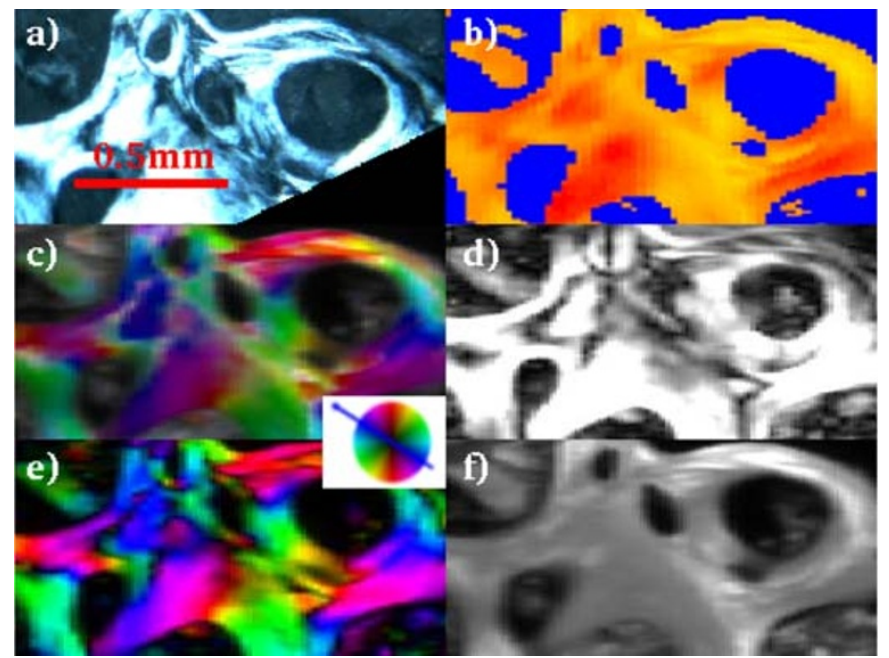

Figure $2 \mid$ CPL (a), WAXS (b), SAXS (c,d,e,f) analyses for the investigated healthy biopsy ( 0.6 is the maximum degree of orientation in $d)$. The colored wheel refers to the SAXS orientation map in panels $\mathbf{c}$ and $\mathbf{e}$.

components of the mineral content whereas the bottom signal is caused by the resin in which the sample has been embedded. This demonstrates how distributions of several individual components can be derived from scanning WAXS data in a quantitative way.

Figs. 2,3,4 show the results of the CPL (image a) and SWAXS (images b-c-d-e-f) analysis for the healthy and pathologic biopsies, respectively.

The CPL (a) image is the map of the collagen fiber orientation within the osteons $\mathbf{s}^{8}$, while the SWAXS information (b,c,d,e,f) refers to the mineral counterpart ${ }^{5,6}$. The main results of the SWAXS data analysis are summarized in the following. In Fig. S2a, besides the blue pattern that corresponds to a background profile (no bone), the red(yellow) 1D-WAXS patterns mainly differ for the sharpness and intensity of the (002) reflections. Both red(yellow) WAXS patterns were fully described by the scattering of the HA nanocrystals, as expected according to ref. 14. The robustness of this analysis is given by the high level of crystallographic information based on several peaks of different width and intensity contained in the analyzed 1D WAXS patterns. As a result of the Rietveld analysis, the selected

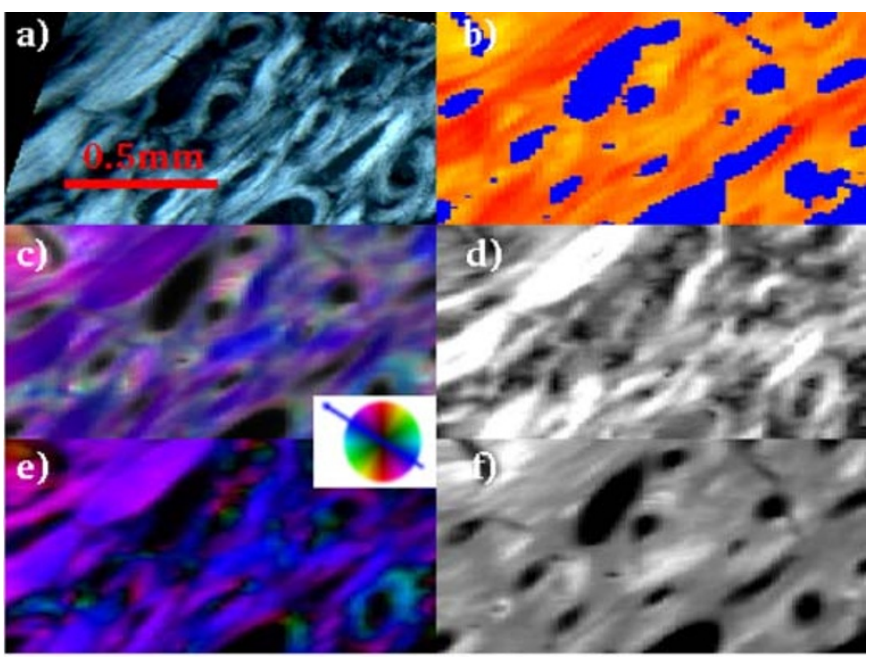

Figure $4 \mid$ CPL (a), WAXS (b), SAXS (c,d,e,f) analyses for the investigated dwarfism - disease biopsy ( 0.8 is the maximum degree of orientation). The colored wheel refers to the SAXS orientation map panels $\mathbf{c}$ and $\mathbf{e}$. 
red(yellow) 1D-WAXS patterns could be described by scattering domains $l=210(88) \AA$ large in the [002] $c$-axis direction, and $w=25(94) \AA$ in the orthogonal [010] direction, respectively. Besides the more pronounced anisotropic shape, a preferred orientation along the [002] crystallographic direction was also included in the modeling of the red pattern (Fig. S2b), in order to correctly describe the sharpness of the (002) reflection. The red pattern with the extremely pronounced (002) peak can be interpreted as being related to mineral nanocrystals uniaxially oriented along the $c$-axis (alias [002] direction) and coherently aligned within the $a b$ plane, i.e., parallel packed nanocrystals ${ }^{18-23}$. The scanning WAXS data were transformed into images showing, for each point, which of the three WAXS patterns is dominating (see WAXS images in the $\mathbf{b}$ panels of Figs. 2,3,4).

The SAXS measurements have been performed at exactly the same positions and were analyzed in an automated way as well ${ }^{11}$. The scattered intensity could be ascribed to the mineral elongated nanocrystals whose elongation direction and orientation degree are represented in the panels $\mathrm{c}$ and d of Figs. 2,3,4, respectively: the higher the degree of orientation, the brighter the intensity in panel $\mathbf{d}$. We additionally plotted also the asymmetric (panel e) and isotropic (panel f) components of figure $\mathrm{c}$. We attribute the asymmetric scattering mainly to the interfibrillar mineralization (preferentially oriented platelets). Concerning the isotropic scattering this could be due to the distribution of the extrafibrillar bone mineralization but also to randomly oriented microfibrils (internally mineralized).

\section{Discussion}

Geometry, orientation and architecture of the mineral nanocrystals within bone are all indirect determinants of the bone strength/ fragility ${ }^{24,25}$.

There is a growing interest in bone biomechanical models at the nanoscale $e^{26,27}$. In this respect, WAXS and SAXS microscopies are promising methods for the creation of computational models capable to represent the organization of mineral nanocrystals and collagen fibers.

Moreover, several bone diseases are related to an abnormal arrangement of the mineral and the collagenic phases at the nanoscale, therefore increasing the interest for bone characterization at such length scale.

In particular, dwarfism can be associated with many different medical conditions, with symptoms and characteristics of the patients varying greatly.

Few evidences were provided about the alterations of collagen orientation and deposition in the pathologies that show symptoms typical of dwarfism. For example, in achondrogenesis of type I the diaphyseal orientation of bone trabeculae and collagen fibers within the trabeculae is disturbed ${ }^{28}$.

In thanatophoric dysplasia, SEM examination showed normal and abnormal bone trabeculae adjacent to each other. In the abnormal trabeculae there are large densely packed osteoblastic and osteocytic lacunae. The calcified collagen fibers have random orientation, in contrast to the longitudinal orientation in the normal bone ${ }^{29}$.

Moreover, some genetic studies based on knock-out mice suggest an important involvement of the bone collagen in the symptoms related to dwarfism. For example, in 35-day-old bcl-2 knockout mice for Bcl-2, that are growth retarded or 'dwarfed', the most marked histological effects of bcl-2 ablation are on osteoblasts and bone. The knockout mice exhibited more disorganized collagen in comparison to the control tissue and with a pseudo-woven appearance ${ }^{30}$.

In another study, mice lacking of Mia3 (Melanoma inhibitory activity member 3 , that is an evolutionarily conserved endoplasmic reticulum resident transmembrane protein), were defective for the secretion of numerous collagens, including collagen I, leading to dwarfism and neonatal lethality. In these mice, collagen deposition was abnormal, and extracellular matrix composition was compromised. These changes were associated with intracellular accumulation of collagen and the induction of a strong unfolded protein response, primarily within the developing skeleton ${ }^{31}$.

Paget's disease is characterized by an increase in size, number, and activity of osteoclasts. The consequent increase in activity of osteoblasts producing new bone results in poor quality tissue, mainly woven with irregular arrangement of collagen fibers ${ }^{32,33}$.

Recently the genetic origin of this disease has been proven, even if a viral trigger is not excluded ${ }^{34}$.

In this panorama, some supporting and some new evidences can be found in the here compared SWAXS and CPL microscopy images, as summarized in the following.

Orientation degree - The bright CPL areas labeled as $\mathrm{T}$ in the osteon classification Table S1, composed of collagen fibers placed transversal to the osteon axis (panels a in Figs. 2,3,4), are spatially correlated with the region of higher orientation degree of the mineral nanocrystals $c$-axis (panels $d$ in Figs. 2,3,4).

Nanocrystal orientation - The red(yellow) WAXS contribution of Fig. 2,3,4b have been identified as HA nanocrystals with and without a preferred orientation along the [002] crystallographic direction, respectively, as described above. A preferred orientation is expected for interfibrillar mineralization ${ }^{35}$. It is known that HA nanocrystals are primarily deposited in the gaps between the collagen fibrils, which work as natural template for HA nanocrystal deposition and preferred orientation. Indeed, the biopsies differ for the abundance of the red/yellow contribution in the WAXS images. However, the lack of preferred orientation in the yellow regions depends only on the actual orientation of the nanocrystals, which is clearly visualized in the scanning SAXS maps of Figs. 2,3,4c. When the nanocrystals were blue(violet) oriented in the scanning SAXS maps of Figs. 2,3,4c, the preferred orientation was registered in the WAXS data. Indeed, as explained in the Materials and Methods section, only a quarter of the entire diffraction ring was registered for the WAXS data. The position of the WAXS detector allowed to register the preferred orientation only for specific positions of the nanocrystals (blue(violet) signal of Figs. 2,3,4c). However, even with this limitation, SWAXS data clearly indicate that the dwarfism disease biopsy has abnormally large areas with mono-oriented aligned interfibrillar mineral nanocrystals, with respect to the other biopsies, as SAXS data are practically dominated by a single orientation. In addition, the higher and lower orientation degree was measured by SAXS for the dwarfism and Paget's disease biopsies, respectively. The Paget case shows more fragmented and disorganized structures. The age difference among patients, namely 27 for the dwarfism disease biopsy and more that 80 years for the others, may play a role.

Another interesting result can be derived from the map of the symmetric component of the SAXS data (panel f of Figs. 2-4). The areas with the highest illumination correspond to sample regions with the mostly isotropically distributed SAXS intensity. This fingerprint is here ascribed to extrafibrillar mineralization, which typically occurs with randomly distributed and randomly oriented HA nanocrystals ${ }^{35}$, or to randomly oriented mineralized microfibrils. Conversely, panels e of Figs. 2,3,4 map only the interfibrillar bone made of unidirectionally oriented nanocrystals.

The fundamental question for many studies performed in the last decades on bone pathologies is how it is possible that a mutation at the molecular/nanoscale level could lead to the failure of the macroscopic tissue. Indeed, answering this question is not trivial, but novel diagnostic systems to image matter at microscopic and nanoscopic resolution are available to offer possible additional information. Here, by inspecting for the first time dwarfism syndrome and Paget's disease biopsies with SWAXS and CPL microscopies, we intend to shed light onto the geometry, orientation and architecture of the mineral nanocrystals, embedded (with and without preferred orientation) in the collagen fibrillar matrix, indirect determinants of the bone strength/fragility and flexibility. Any clinical interpretation 
of these findings is beyond the main purpose of the present paper, which aims at presenting the results of this quantitative imaging method, based on the combination of SWAXS and CPL microscopy images, to map the actual orientation of HA nanocrystals across healthy and pathologic biopsies. The presented method allows to visualize and distinguish between interfibrillar bone mineralization (preferred orientation) and other contributions.. Although the orientation of the collagen and mineral particles is tridimensional, and a complete quantitative texture analysis ${ }^{36-37}$ is not possible based on the 2D SWAXS measurements here recorded, clear differences in the texture of the investigated samples are recognized, demonstrating the effectiveness of the proposed approach in the nanoscale studies of the bone pathologies. In addition, this information enables clarifying the models generally associated with polarized light observations.

Among the samples investigated in this study, the dwarfism specimen was outstanding both in degree and uniformity of the orientation of the interfibrillar mineral phase. The Paget's disease sample had the lowest degree and largest variety of orientations, and the sample without any musculoskeletal disease ranged in between these two extremes. The expected profound impact of these differences in the nanostructure, on the performance of the bones, should be studied both in future fracture studies and via modeling.

\section{Methods}

Biopsies for the Paget's disease and healthy bones were obtained from patients having approximately the same age, of 83 and 85 years. Only the dwarfism affected bone was collected from a 27 years old man.

Femur neck pieces were retrieved from surgery and fixed in buffered formalin at $4 \%$ until testing. Each femur neck slice was cut to $100 \pm 20 \mu \mathrm{m}$ thickness and embedded in polymethylmethacrylate (Carlo Erba Reagenti SpA, Rodano, Milano, Italy) for CPL and SWAXS measurements. Written informed consent was obtained from patients. The informed consent procedure applied at the Rizzoli Orthopaedic Institute is in compliance with the Italian and European rules for the collection of tissue from human donors.

For CPL, each slice was visualized at $4 \mathrm{x}$ objective lens magnification and $10 \mathrm{x}$ ocular lens magnification by polarized circular light microscope (Leica DM EP) and classified according to specifications in Table S1.

SWAXS microscopy. Proposal on 'Scanning SAXS \& WAXS analysis of osteonal collagen fiber orientation in human cortical bone. Effect of different skeletal diseases' was approved by the SLS Program Review Committees (Proposal ID: 20100956). SWAXS experiments were performed at the cSAXS beamline ${ }^{11}$ of the Swiss Light Source (SLS), Paul Scherrer Institut, Villigen, Switzerland, in accordance with all relevant regulations including the Swiss ordinance 814.912 on the contained use of organisms. A monochromatic X-ray beam $(\mathrm{E}=18.58 \mathrm{keV})$ was focused down to about $30 \times 5 \mu \mathrm{m}^{2}$ by a bend monochromator crystal and a bend mirror. This sample is raster scanned through this focal spot, with the detector measuring the total intensity integrated over the exposure time coming from the currently illuminated spot on the sample. The image is calculated from these data when the scanning process is finished and data reduction/analysis performed.

To speed up acquisition, data have been recorded in a continuous line scan mode with the sample moving at constant speed along a line of the $2 \mathrm{D}$ raster scan $(\mathrm{X}-\mathrm{Y})$ while the Pilatus detector is continuously recording data. SAXS and WAXS data were collected at sample-detector distances of 2158 and $613 \mathrm{~mm}$, respectively. The detector position was roughly centered with respect to the direct beam only for the SAXS data acquisition while it was translated to have the direct beam in the left bottom corner during WAXS data collection. Therefore WAXS data contain only a quarter of the whole diffraction ring.

Additional scanning SAXS measurements were done using the XMI-LAB equipment at the IC-CNR, in order to further complement and support the results from cSAXS beam line by investigating larger areas of the samples. The XMI-LAB @ IC$\mathrm{CNR}$ is equipped with a synchrotron class rotating anode X-ray microsource (Rigaku FR-E + SuperBright $\left(4^{*} 10^{9}\right.$ photons $\left./ \mathrm{sec} / \mathrm{mm}^{2} / \mathrm{mR}^{2}, 2475 \mathrm{~W}\right)$ coupled through a focusing high-flux multilayer optics (Cu radiation) to a three-pinhole camera (SMAX-3000). The minimal focal spot at the sample position (circular shape) is $\sim 0.07 \times 0.07 \mathrm{~mm}^{2}$. The system is equipped with two distinct $2 \mathrm{D}$ detectors: Triton ${ }^{\mathrm{TM}} 20$ gas-filled proportional counter SAXS detector, $20 \mathrm{~cm}$ in diameter and $200 \mu \mathrm{m}$ pixel size, and RAXIA image plate WAXS detector with off-line read-out unit and $100 \mu \mathrm{m}$ pixel size. The WAXS detector has a $1 \mathrm{~cm}$ hole in the center to leave the direct beam passing through for the simultaneous acquisition of SAXS and WAXS data.

In the entire raster scanned area, the SAXS and WAXS 2D images registered at cSAXS were azimuthally integrated to $1 \mathrm{D}$ patterns. Given the great amount of data, a finite and small number (say N) of 1D patterns were selected for each sample, by applying an 'adaptive binning' criterion ${ }^{12}$. The first patterns to be selected were the WAXS ones. In order to pick up the less correlated M patterns (the "signals"), we found the global minimum of $\left(\mathrm{N}^{\mathrm{M}}\right)$-dimensional covariance matrix, obtained by symmetrizing the original $2 \mathrm{D}(\mathrm{N} \times \mathrm{N})$ covariance matrix in a $\left(\mathrm{N}^{\mathrm{M}}\right)$-dimensional space. In our case we used $\mathrm{N}=61$ and $\mathrm{M}=3$. For each of the three selected 1D WAXS patterns, we extracted the corresponding 1D SAXS data set, collected exactly at the same sample position. Fig. S2a shows the selected 1D-WAXS patterns found through the 'adaptive binning' criterion ${ }^{12}$, for the healthy case. Similar profiles were found for each sample.

1D WAXS and SAXS patterns analysis. The selected 1D WAXS and SAXS patterns, representing the major components of the whole experimental data set, were analyzed by suitable crystallographic approaches.

Precisely, the 1D WAXS patterns were analyzed by means of a whole profile fitting Rietveld-based program, named FULLPROF, taking into account crystal structure models and possible shape anisotropies ${ }^{13}$. The 1D WAXS patterns were fully described by HA nanocrystals according to ${ }^{14}$. The background was linearly interpolated and not refined. The anisotropic peak broadening was described by a phenomenological model based on a modified Scherrer formula. The anisotropic peak broadening accounts for the crystal shape anisotropy (elongated shape). The relevant output data of the fits are the dimensions of the elongated scattering domain (length $l$ and width $w$ ). Fig. S2b shows the results of a typical fit, which corresponds to the red pattern in Fig. S2a.

The 1D-SAXS patterns, selected in correspondence of the 1D-WAXS patterns, were interpreted using a crystallographic software for SAXS data analysis ${ }^{15}$.

A scattering object, modeled by a cylinder of length $L$ and radius $R$, has a radius of gyration $(\mathrm{Rg})$, describing the mass distribution of the object around its center of gravity, which can be evaluated as:

$$
R_{g}=\sqrt{\frac{L^{2}}{12}+\frac{R^{2}}{2}}
$$

The radius of gyration was extracted for each of the analyzed 1D SAXS patterns and compared with the Rg value expected for the $\mathrm{L}$ and $\mathrm{R}$ scattering domain size evaluated by the WAXS data analysis.

Fig. S3 shows the results of a typical fit: the fit (right figure), and the corresponding pair distribution function (left figure) are shown. The value extracted from the fit for the radius of gyration, $\mathrm{Rg}=61.4 \AA$, agrees very well with the value of $61.3 \AA \mathrm{cal}-$ culated from eq. (1) using the scattering domain size ( $\mathrm{L}=l=210 \AA, 2 * \mathrm{R}=w=25 \AA$ ) found from the Rietveld analysis of the corresponding 1D-WAXS pattern in Fig. S2b This cross check between the analysis of the 1D SAXS and WAXS patterns was repeated on all analyzed 1D-SAXS and WAXS patterns and proved that the measured intensity is dominated by the scattering of mineral nanocrystals of elongated shape both in WAXS and in SAXS measurements, in agreement with ref. 5-6. Indeed, SWAXS microscopy images provide mainly information on the mineral nanocrys talline structure and morphology. The 1D SAXS analysis yielded also the $\Delta \mathrm{q}$ range for the scanning SAXS data analysis described below.

The previously described analysis allowed us to attribute sample features to the selected 1D WAXS and SAXS patterns. Maps of the distribution of these major scattering components (scanning WAXS and SAXS), spatially resolved over the sample, reveal thereby quantitatively the distribution of these relevant sample features.

The scanning WAXS image in the flow chart of Fig. S1 was elaborated by using a statistical technique, called Canonical Correlation Analysis (CCA). CCA is a multichannel generalization of ordinary correlation analysis, which quantifies the relationship between two random variables $\mathrm{x}$ and $\mathrm{y}$ by means of the so-called correlation coefficient, a scalar quantity with a value between -1 and 1 , that measures the degree of linear dependence between $\mathrm{x}$ and $\mathrm{y}^{16}$. When applying correlation analysis to WAXS data, the variables $\mathrm{x}$ and $\mathrm{y}$ need to be specified. In ordinary correlation analysis, $\mathrm{x}$ and $\mathrm{y}$ are univariate variables and, specifically, the $\mathrm{x}$ variable consists of the intensity spectrum of the measured 1D WAXS pattern contained in each pixel, whereas the $y$ variable consists of a model intensity spectrum ${ }^{17}$, either the pure phase pattern, or a theoretical mixture profile. Here, the set of model spectra is shown in Fig. S2a for the healthy biopsy. Similar models were extracted, using the adaptive binning method, for the other biopsies. The colored scanning WAXS image indicates, pixel by pixel, where each of the three 1D WAXS selected patterns are represented in the XY rastered area (reported in Fig. 1).

The scanning SAXS image in the flow chart of Fig. S1 was elaborated using the multimodal $\mathrm{x}$-ray scattering imaging method $^{11}$, which allows to analyze the anisotropy of the SAXS scattering and, from it, to evaluate the predominant orientation of the crystal, which is perpendicular to the orientation of the scattered intensity. In order to further clarify the SAXS analysis, an elongated object is shown in Fig. S4a (direct space) of the Supplementary Information. This object will scatter an equivalently elongated intensity distribution (elongation c axis), shown in Fig. S4b, which is just turned by 90 degrees in the reciprocal space. The multimodal x-ray imaging method allowed us to analyze, image by image, the intensity distribution anisotropy and extract quantitatively the main elongation direction of the scattering object (Fig. S4c) and the orientation degree (Fig. S4d). The first step of the SAXS analysis is the azimuthal integration.

To capture the asymmetry of the scattering pattern, the integration is performed in 16 azimuthal segments. The intensity is normalized by the number of pixels in the segment covering the radial and the azimuthal range. The resulting intensity distribution, for typical SAXS samples with moderate structural ordering and inversion symmetry of the SAXS signal, is well approximated by a sinusoidal function and then the parameters for degree of orientation and the orientation of the average scattered 
intensity can be extracted from the discrete Fourier transform of the above integrated intensities. Precisely, within a certain q-range (length scale), selected from the previous $1 D$ - WAXS and SAXS analysis, 'symmetric' $a_{0}$ and 'asymmetric' intensity $a_{1}$ are evaluated for each SAXS data frame: $\mathrm{a}_{0}$ is the average intensity within the q-range, and $a_{1}$ is the amplitude of the sinusoidal intensity variation with respect to $a_{0}$. The degree of orientation, defined as $a_{1} / a_{0}$, ranges therefore from 0 to 1 , i.e., from 0 to $100 \%$ oriented.

1. Werner, S. \& Wagner, H. D. The Material Bone: Structure-Mechanical Function Relations. Annu. Rev. Mater. Res. 28, 271-298 (1998).

2. Rho, J. Y., Kuhn-Spearing, L. \& Zioupos, P. Mechanical properties and the hierarchical structure of bone. Med. Eng. \& Phys. 20, 92-102 (2002).

3. Olszta, M. J., Cheng, X., Jee, S. S., Kumar, R., Kim, Y. Y. \& Kaufman, M. J. et al. Bone structure and formation: A new perspective. Mat. Sci. \& Eng. R 58, 77-116 (2007).

4. Dunlop, J. D. W. \& Fratzl, P. Biological Composites. Annu. Rev. Mater. Res. 40, 124 (2010).

5. Paris, O. From diffraction to imaging: New avenues in studying hierarchical biological tissues with x-ray microbeams - Review. Biointerphases 3(2), FB16FB26 (2008).

6. Fratzl, P. Bone mineralization density distribution in health and disease, - Review. Bone 42,456-466 (2008).

7. Dorfs, D., Krahne, R., Giannini, C., Falqui, A., Zanchet, A. \& Manna, L. Synthesis and characterization of quantum dots. In: Andrews D, Scholes G, Wiederrecht G editors. Comprehensive Nanoscience and Technology Elsevier, p. 219-270 (2011)

8. Beraudi, A. Stea, S. Bordini, B. Baleani, M. \& Viceconti, M. Osteon Classification in Human Fibular Shaft by Circularly Polarized Light. Cells Tissues Organs 191(3), 260-268 (2010).

9. Feng, X. \& McDonald, J. M. Disorders of Bone modeling. Annu. Rev. Mater. Res. 6 , 121-125 (2011).

10. Adler, C. P. Bone diseases: macroscopic, histological, and radiological diagnosis of structural changes in the skeleton. Springer -Verlag, Berlin - Heidelberg (2000).

11. Bunk, O. et al. Multimodal x-ray scatter imaging. New Journal of Physics 11, 123016 (2009).

12. Guagliardi, A. et al. Canonical correlation and quantitative phase analysis of microdiffraction patterns in bone tissue engineering. J Appl Cryst 40, 865-873 (2007).

13. http://www-llb.cea.fr/fullweb/ (accessed on the 8th of May, 2012).

14. Kay, M. I., Young, R. A. \& Posner, A. S. Crystal structure of hydroxyapatite. Nature 25, 1050 (1964).

15. http://www.embl-hamburg.de/biosaxs/manual_gnom.html (accessed on the $8^{\text {th }}$ of May, 2012)

16. Laudadio, T., Pels, P., De Lathauwer, L., Van Hecke, P. \& Van Huffel S. Tissue Segmentation and Classification of MRSI Data Using Canonical Correlation Analysis. Magn Reson Med. 54, 1519-1529 (2005).

17. Ladisa, M., Lamura, A. \& Laudadio, T. Classification of Crystallographic Data Using Canonical Correlation Analysis, EURASIP. J Adv Sign Proc 2007, 19260(18) (2007).

18. Landis, W. J., Song, M. J., Leith, A., McEwen, L. \& McEwen, B. F., Mineral and organic matrix interaction in normally calcifying tendon visualized in 3 dimensions by high-voltage electron-microscopic tomography and graphic image-reconstruction. J Struct Biol 110 (1), 39-54 (1993).

19. Landis, W. J. et al. Mineralization of collagen may occur on fibril surfaces: evidence from conventional and high-voltage electron microscopy and threedimensional imaging. J Struct Biol 117(1), 24-35 (1996).

20. Rhee, S. H., Rhee, S. H., Suetsugu, Y. \& Tanaka, J. Biomimetic configurational arrays of hydroxyapatite nanocrystals on bio-organics. Biomaterials 22, 28432847 (2001).

21. Traub, W. Arad, T. \& Weiner, S. 3-Dimensional ordered distribution of crystals in turkey tendon collagen-fibers. Proc Natl Acad Sci. U.S.A. 86, 9822-9826 (1989).

22. Landis, W. J. The strength of a calcified tissue depends in part on the molecularstructure and organization of its constituent mineral crystals in their organic matrix. Bone 16, 533-544 (1995).

23. Weiner, S., Arad, T. \& Traub, W. Crystal organization in rat bone lamellae. FEBS Lett 285, 49-54 (1991).

24. Ruppel, M. E., Miller, L. M. \& Burr, D. B. The effect of the microscopic and nanoscale structure on bone fragility. Osteopros Int 19, 1251-1265 (2008).
25. Wang, R. \& Gupta, H. S. Deformation and Fracture Mechanism of Bone and Nacre. Annu Rev Mater Res 41, 41-73 (2011).

26. Reisinger, A. G., Pahr, D. H. \& Zysset, P. K. Principal stiffness orientation and degree of anisotropy of human osteons based on nanoindentation in three distinct planes. J Mech Behav Biomed Mater 4(8), 2113-27 (2011).

27. Webster, D. \& Müller, R. In silico models of bone remodeling from macro to nanofrom organ to cell. Wiley Interdisciplinary Reviews-Systems Biology And Medicine 3 (2), 241-251 (2011)

28. Ornoy, A., Sekeles, E., Smith, P., Simkin, A. \& Kohn, G. Achondrogenesis type I in three sibling fetuses. Scanning and transmission electron microscopic studies. Am J Pathol 82(1), 71-84 (1976).

29. Ornoy, A., Adomian, G. E., Eteson, D. J., Burgeson, R. E. \& Rimoin, D. L. The role of mesenchyme-like tissue in the pathogenesis of thanatophoric dysplasia. Am J Med Genet. 21(4), 613-30 (1985).

30. Boot-Handford, R. P. et al. The bcl-2 knockout mouse exhibits marked changes in osteoblast phenotype and collagen deposition in bone as well as a mild growth plate phenotype. Int J Exp Path 79, 329-335 (1998).

31. Wilson, D. G. et al. Global defects in collagen secretion in a Mia3/TANGO1 knockout mouse. J Cell Biol 193(5), 935-51 (2011).

32. Misra, D. P. Crosslink in bone collagen in Paget's disease. J Clin Pathol 28(4), 3058 (1975).

33. Garnero, P. Gineyts, E. Schaffer, A. V. Seaman, J. \& Delmas, P. D. Measurement of urinary excretion of nonisomerized and beta-isomerized forms of type I collagen breakdown products to monitor the effects of the bisphosphonate zoledronate in Paget's disease. Arthritis Rheum 41(2),354-60 (1998).

34. Michou, L. \& Brown, J. P. Genetics of bone diseases: Paget's disease, fibrous dysplasia, osteopetrosis, and osteogenesis imperfecta. Joint Bone Spine 78(3), 2528 (2011).

35. Reisinger, A. G., Pahr, D. H. \& Zysset, P. K. Sensitivity analysis and parametric study of elastic properties of an unidirectional mineralized bone fibril-array using mean field methods. Biomech Model Mechanobiol 9, 499-510 (2010).

36. Wenk, H.-R. and Heidel Crystal Alignment of Carbonated Apatite in Bone and Calcified Tendon: Results from Quantitative Texture Analysis. Bone 24 (4), 361369 (1999).

37. Wagermaier, W. et al. Scanning texture analysis of lamellar bone. J. Appl. Cryst. 40, 115-120 (2007)

\section{Acknowledgments}

This work has been partially supported by the SEED project "X-ray synchrotron class rotating anode microsource for the structural micro imaging of nanomaterials and engineered biotissues (XMI-LAB)"- IIT Protocol n.21537 of 23/12/2009. The SAXS/WAXS experiments were performed on the cSAXS beamline at the Swiss Light Source, Paul Scherrer Institut, Villigen, Switzerland. Mr R. Lassandro is acknowledged for his full technical support with the XMI-LAB laboratory, where additional SAXS and WAXS measurements were acquired in support to the work.

\section{Author contributions}

C.G., F.B., D.S. and O.B. conceived and realized the SWAXS experiment at cSAXS-PSI, A.B prepared the biopsies, acquired and interpreted the CPL images, D.A. complemented the SAXS/WAXS measurements at the XMI-LAB. M.L. dealt with SWAXS data reduction (adaptive binning, CCA). D.S., M.L., O.B. and C.G. transformed the SWAXS raw data into quantitative radiographies. C.G. discussed the results and wrote the paper in close collaboration with all the authors.

\section{Additional information}

Supplementary information accompanies this paper at http://www.nature.com/ scientificreports

Competing financial interests: The authors declare no competing financial interests.

License: This work is licensed under a Creative Commons

Attribution-NonCommercial-ShareAlike 3.0 Unported License. To view a copy of this license, visit http://creativecommons.org/licenses/by-nc-sa/3.0/

How to cite this article: Giannini, C. et al. Correlative Light and Scanning X-Ray Scattering Microscopy Of Healthy and Pathologic Human Bone Sections. Sci. Rep. 2, 435; DOI:10.1038/srep00435 (2012) 